Luis Felipe Chaga Maronezi

Ohttp://orcid.org/0000-0001-7947-939x

Giovana Bonessoni Felizari Ohttp://orcid.org/0000-0002-1408-6285

Guilherme Assoni Gomes Ohttp://orcid.org/0000-0003-2998-0877

Jeanice de Freitas Fernandes O http://orcid.org/0000-0003-2137-7336

Rogério Tomasi Riffel Ohttp://orcid.org/0000-0002-2285-204X

Ivana Loraine Lindemann (Dhtp://orcid org/0000-0002-6222-9746

\section{Prevalência e características das violências e intoxicações exógenas autoprovocadas: um estudo a partir de base de dados sobre notificações}

\author{
Prevalence and characteristics of self-inflicted exogenous violence \\ and intoxication: a study from a database on notifications
}

DOI: $10.1590 / 0047-2085000000349$

\section{RESUMO}

Objetivo: Descrever os casos autoprovocados de violência e de intoxicação exógena aguda ocorridos no Rio Grande do Sul (RS) entre os anos de 2013 e 2017. Métodos: Estudo transversal incluindo todos os casos notificados no Sistema de Agravos de Notificação (Sinan) entre 2013 e 2017, selecionados por meio das fichas de violência interpessoal/autoprovocada, por meio da variável lesão provocada, e de investigação de intoxicação exógena, pela variável circunstância da exposição/contaminação. A análise compreendeu a estatística descritiva, o cálculo das prevalências por 100.000 habitantes e a estratificação por sexo conforme características sociodemográficas e clínicas. Resultados: No período proposto, foram notificados 18.544 casos autoprovocados de violência e 5.624 de intoxicação exógena, com predomínio de mulheres (67\% e 75,3\%), entre 30 e 59 anos (46,4\% e 49\%), brancas (86\% e $86 \%$ ), com ensino fundamental completo/incompleto (58,3\% e 47,3\%), residentes em zona urbana/ periurbana (89,2\% e 89,6\%) e com episódios ocorridos na própria residência (88,8\% e 96,6\%). Quanto aos métodos de violência empregados, destacaram-se os envenenamentos/intoxicações (39,7\%), enforcamentos (13,8\%), agentes perfurocortantes $(13,6 \%)$ e armas de fogo $(2,0 \%)$. Verificou-se diferença conforme o sexo nas variáveis sociodemográficas e clínicas referentes a ambos os agravos, bem como um aumento de 169\% e 381,1\%, respectivamente, nas prevalências de violência e intoxicação entre os anos de 2013 e 2017. Conclusões: Evidenciou-se um expressivo e crescente número de casos no estado, com maiores implicações no gênero feminino, importantes diferenças de acordo com sexo e populações mais acometidas, fomentando a necessidade da implementação de medidas preventivas específicas nos grupos vulneráveis.

\section{PALAVRAS-CHAVE}

Sistemas de informação, doenças e agravos de notificação compulsória, tentativa de suicídio, violência, saúde pública.

\section{ABSTRACT}

Objective: To describe the self-inflicted cases of violence and acute exogenous intoxication occurred in the state of Rio Grande do Sul (RS) between 2013 and 2017. Methods: Cross-sectional study including all cases reported in the Notifiable Diseases System between 2013 and 2017, selected through the forms of interpersonal/self-infliged, through the variable caused injury, and through the investigation of exogenous intoxication, due to the variable circumstances of exposure/contamination. The analysis included descriptive statistics, calculation of prevalences per 100.000 and stratification by sex according to sociodemographic and clinical characteristics. Results: In the proposed period, 18.544 cases of violence and 5.624 cases of exogenous intoxication self-infliged were reported, with a predominance of women (67\% and 75,3\%), between 30 and 59 years old (46,4\% and $49 \%)$, white ( $86 \%$ and $86 \%)$, with complete/incomplete elementary education (58,3\% and 47,3\%), living in urban/periurban areas (89,2\% and $89,6 \%$ ) and with episodes occurring at their own residence $(88,8 \%$ and $96,6 \%)$. About the methods of violence used, poisonings/intoxications (39,7\%), hangings (13,8\%), sharp objects (13,6\%) and firearms (2\%) stood out. There was a difference according to sex in the sociodemographic and clinical variables referring to both diseases, as well as an increase of $169 \%$ and $381,1 \%$ in prevalences of violence and intoxication, between 2013 and 2017. Conclusions: An expressive and growing number of cases in the state, with greater implications for females, important differences according to sex and most affected populations, encourages the need to implement specific preventive measures in vulnerable groups.

KEYWORDS

Information systems, disease notification, suicide attempted, violence, public health.

Received in: Jan/24/2021. Approved in: Aug/5/202

1 Universidade Federal da Fronteira Sul, Passo Fundo, RS, Brasil.

2 Universidade de Passo Fundo, Passo Fundo, RS, Brasil.

Address for correspondence: Luis Felipe Chaga Maronezi. Rua Paissandu, 1932, Ap 703 - 99010-102 - Passo Fundo, RS, Brasil E-mail: luisfelipemaronezi@hotmail.com 


\section{INTRODUÇÃO}

O grupo das violências autoprovocadas engloba situações de ideação suicida, autoagressão, tentativa de suicídio e suicídio consumado, representando um fenômeno complexo e multicausal, associado a fatores psicossociais, emocionais, de gênero e de aceitabilidade sociocultural das vítimas'.

Quanto às intoxicações exógenas, podem ser definidas como um desequilíbrio orgânico causado pela administração de substâncias químicas nocivas ao organismo e, frequentemente, são um meio utilizado em casos de violência autoprovocada, representando cerca de $70 \%$ dos casos de tentativa de suicídio notificados no Brasil. Entre as principais substâncias utilizadas, estão os agrotóxicos, com maiores frequências nos países em desenvolvimento, e os medicamentos, mais comuns em países desenvolvidos².

A fronteira entre autonegligência, lesão autoprovocada, ideação suicida, tentativa e suicídio efetivo é tênue e é consenso que a morte autoinfligida é pensada, preparada e, muitas vezes, antecedida por tentativas, caracterizando-se, portanto, em grande parte das vezes, como uma causa de morte evitável e prevenível ${ }^{3}$.

Em 2015 foram registrados mundialmente 12 casos de suicídio para cada 100.000 habitantes, colocando a causa em 14a posição em relação à mortalidade, e foram apontados como principais métodos os pesticidas, o enforcamento e as armas de fogo'. O Brasil desponta entre os 10 países com maior número de óbitos pela causa (6/100.000 habitantes/ano); ${ }^{4}$ no estado do Rio Grande do Sul se observam elevadas taxas em comparação às demais unidades federativas, com registro, entre 2005 e 2013, de 34.166 tentativas de suicídio por autoenvenenamento, representando taxa média anual de 36 a cada 100.000 habitantes, números que vêm preocupando autoridades e entidades da saúde $e^{1,2,5}$.

Conforme a Portaria no 204 do Ministério da Saúde, todas as violências autoprovocadas e as intoxicações exógenas são de notificação compulsória e imediata desde o ano de 2016. $\mathrm{O}$ ato é obrigatório para médicos, profissionais de saúde ou responsáveis por serviços públicos e privados de saúde que prestem assistência aos pacientes, aplicando-se tanto para os casos suspeitos quanto para os confirmados, devendo, obrigatoriamente, ser realizado nas primeiras 24 horas após o ocorrido ${ }^{6,7}$. Contudo, todas as situações são subnotificadas mesmo em países com bons sistemas de informação e, segundo a Organização Mundial da Saúde, existem evidências de que apenas 25\% dos indivíduos que tentam suicídio entram em contato com algum sistema de saúde, dificultando o acesso a informações fidedignas acerca das ocorrências ${ }^{3}$.

Ainda assim, o controle mais efetivo dessas violências depende da identificação, da correta classificação e da notificação dos casos, de modo que, ao caracterizar a demanda, a descrição das populações acometidas pode fornecer instrumentos importantes para a adoção de estratégias de prevenção, rastreamento, monitoramento e intervenção, especialmente no âmbito da saúde pública e da atenção primária à saúde ${ }^{8}$.

Nesse sentido, o presente estudo buscou descrever a distribuição dos casos autoprovocados, com óbito não consumado, de violências e de intoxicações exógenas agudas notificados nos municípios do estado do Rio Grande do Sul entre os anos de 2013 e 2017, bem como delinear as características comuns às vítimas.

\section{MÉTODOS}

Trata-se de um estudo transversal realizado com todos os casos autoprovocados de violência e de intoxicação exógena notificados no Sistema de Informação de Agravos de Notificação (Sinan) do Rio Grande do Sul, entre $1^{\circ}$ de janeiro de 2013 e 31 de dezembro de 2017, sem a aplicação de qualquer critério de exclusão.

A seleção dos casos foi feita por meio das notificações realizadas por meio da ficha de violência interpessoal/autoprovocada (CID 10 Y09 - agressão por meios não especificados) e da ficha de investigação de intoxicação exógena (CID 10 T65.9 - efeito tóxico de substância não especificada). A escolha dos episódios autoprovocados foi feita pela variável lesão autoprovocada na ficha de violência e pela variável circunstância da exposição/contaminação na ficha de intoxicação; contudo, salienta-se que um mesmo paciente, na vigência de uma intoxicação autoprovocada, pode ser notificado concomitantemente por meio de ambas as fichas ${ }^{9}$. Apesar de as informações constarem em ambas as fichas, foram consideradas como um só evento para fins de cálculo da prevalência. Destaca-se, ainda, que os óbitos consumados no momento das ocorrências não são notificados via Sinan e, portanto, não foram incluídos no estudo. Os dados foram solicitados ao Centro Estadual de Vigilância em Saúde - Núcleo de Vigilância das Doenças e Agravos Não Transmissíveis e recebidos em formato de planilha eletrônica, com supressão de dados de identificação dos indivíduos.

A análise compreendeu a estatística descritiva (frequências absolutas e relativas das variáveis) e o cálculo da prevalência dos casos por 100.000 habitantes, por ano de ocorrência e por Coordenadoria Regional de Saúde (CRS). O estado do Rio Grande do Sul é dividido, político-administrativamente, em 19 CRSs, responsáveis pelo planejamento, acompanhamento e gerenciamento das ações e serviços de saúde de modo regionalizado. A caracterização da amostra se deu pelas informações constantes nas fichas, englobando sexo, idade, cor de pele, escolaridade, zona de residência e local de ocorrência.

Características exclusivas dos casos de violência foram analisadas e incluíram o método empregado no ato: enforcamento, uso de agentes perfurocortantes, envenenamento/ intoxicação e arma de fogo. Quanto às particularidades clínicas dos casos de intoxicação, foram analisados tipo de agente 
tóxico utilizado (medicamentos, venenos e outros), via de exposição (digestiva e outras), tipo de exposição (aguda única, aguda repetida e crônica), tipo de atendimento (hospitalar e ambulatorial ou domiciliar), necessidade de hospitalização, classificação final (intoxicação confirmada, exposição, reação adversa, diagnóstico diferencial e outros), critérios de confirmação do quadro (clínico, clínico epidemiológico e clínico laboratorial) e evolução do caso (cura sem sequela, óbito e outros). A estratificação por sexo de acordo com características sociodemográficas, método de violência e particularidades clínicas foi determinada pelo teste do qui-quadrado (admitindo-se erro a de 5\%, sendo considerados significativos valores de $p<0,05$, para testes bicaudais).

O estudo foi realizado em consonância com a Resolução no 466/2012 do Conselho Nacional de Saúde, sendo aprovado pelo Comitê de Ética em Pesquisa com Seres Humanos da Universidade Federal da Fronteira Sul (Parecer n 3.189.541) e pelo Comitê de Ética da Escola de Saúde Pública, Secretaria Estadual de Saúde do Rio Grande do Sul (instituição coparticipante, sob o Parecer n॰ 3.257.379).

\section{RESULTADOS}

No período analisado, foram notificados 18.544 casos de violência autoprovocada e a tabela 1 demonstra a caracterização das vítimas. Verificou-se predomínio do sexo feminino (67\%), idade entre 30 e 59 anos (46,4\%), cor da pele branca (86\%), ensino fundamental completo/incompleto $(58,3 \%)$ e residência em zona urbana/periurbana (89,2\%), com a maioria dos episódios ocorridos na própria residência do indivíduo (88,8\%). Quanto aos métodos empregados, destacam-se os envenenamentos/intoxicações (39,7\%), enforcamentos (13,8\%), uso de agentes perfurocortantes $(13,6 \%)$ e armas de fogo (2\%).

Tabela 1. Casos de violência autoprovocada: caracterização e estratificação por sexo. Notificações no estado do Rio Grande do Sul, $2013-2017$ (n=18.544)

\begin{tabular}{|c|c|c|c|c|}
\hline \multirow{2}{*}{ Variáveis } & Total & Masculino & Feminino & \multirow{2}{*}{$\mathbf{p}^{*}$} \\
\hline & n (\%) & $\mathrm{n}(\%)$ & n (\%) & \\
\hline Total & $18.544(100,0)$ & $6.119(33,0)$ & $12.425(67,0)$ & \\
\hline Idade (anos completos) & & & & $<0,01$ \\
\hline$\leq 16$ & $2.787(15,5)$ & $636(10,4)$ & $2.151(17,3)$ & \\
\hline $17-29$ & $5.945(31,5)$ & $2.025(33,1)$ & $3.920(31,5)$ & \\
\hline $30-59$ & $8.602(46,4)$ & $2.803(45,8)$ & $5.799(46,7)$ & \\
\hline$\geq 60$ & $1.210(6,6)$ & $655(10,7)$ & $555(4,5)$ & \\
\hline Cor da pele $(n=17.788)$ & & & & 0,91 \\
\hline Branca & $15.292(86,0)$ & $5.048(86,0)$ & $10.244(85,9)$ & \\
\hline Outras & $2.496(14,0)$ & $821(14,0)$ & $1.675(14,1)$ & \\
\hline Escolaridade $(n=12.616)$ & & & & $<0,01$ \\
\hline Analfabetismo & $182(1,5)$ & $96(2,4)$ & $86(1,0)$ & \\
\hline Ensino fundamental completo/incompleto & $7.355(58,3)$ & $2.460(62,5)$ & $4.895(56,4)$ & \\
\hline Ensino médio incompleto ou mais & $5.079(40,2)$ & $1.382(35,1)$ & $3.697(42,6)$ & \\
\hline Zona de residência (n=17.943) & & & & $<0,01$ \\
\hline Urbana/periurbana & $16.007(89,2)$ & $5.113(86,7)$ & $10.894(90,5)$ & \\
\hline Rural & $1.936(10,8)$ & $786(13,3)$ & $1.150(9,5)$ & \\
\hline Local de ocorrência (n=17.840) & & & & $<0,01$ \\
\hline Própria residência & $15.838(88,8)$ & $4.885(83,3)$ & $10.953(91,5)$ & \\
\hline Outros & $2.002(11,2)$ & $982(16,7)$ & $1.020(8,5)$ & \\
\hline Enforcamento ( $n=18.003$ ) & & & & $<0,01$ \\
\hline $\operatorname{Sim}$ & $2.493(13,8)$ & $1.570(26,4)$ & $923(7,7)$ & \\
\hline Não & $15.510(86,2)$ & $4.384(73,6)$ & $11.126(92,3)$ & \\
\hline Agentes perfurocortantes $(n=17.966)$ & & & & 0,88 \\
\hline $\operatorname{Sim}$ & $2.442(13,6)$ & $803(13,5)$ & $1.639(13,6)$ & \\
\hline Não & $15.524(86,4)$ & $5.129(86,5)$ & $10.395(86,4)$ & \\
\hline Envenenamento/intoxicação ( $\mathrm{n}=18.046)$ & & & & $<0,01$ \\
\hline $\operatorname{Sim}$ & $7.166(39,7)$ & $1.801(30,3)$ & $5.365(44,3)$ & \\
\hline Não & $10.880(60,3)$ & $4.142(69,7)$ & $6.738(55,7)$ & \\
\hline Arma de fogo $(n=17.946)$ & & & & $<0,01$ \\
\hline $\operatorname{Sim}$ & $366(2,0)$ & $279(4,7)$ & $87(0,7)$ & \\
\hline Não & $17.580(98,0)$ & $5.645(95,3)$ & $11.935(99,3)$ & \\
\hline
\end{tabular}

* Teste do qui-quadrado.

Fonte: Sistema de Informação de Agravos de Notificação (SINAN). 
Ainda na tabela 1, verificam-se as distribuições das características das notificações conforme o sexo dos indivíduos, observando-se diferença estatisticamente significativa conforme idade, escolaridade, zona de residência e local de ocorrência dos casos. Identificou-se que mulheres de idade mais jovem (menor ou igual a 16 anos) apresentaram frequências maiores que os homens da mesma faixa etária, do mesmo modo que homens com mais de 60 anos apresentaram número de casos superior às mulheres de mesma idade $(p<0,01)$. Em comparação aos homens, foi observada maior frequência em mulheres com escolaridade mais elevada, habitação em zona urbana ou periurbana e ocorrências na própria residência $(p<0,01)$. Também se encontrou diferença no método empregado na violência, predominando o uso de armas de fogo e práticas de enforcamento em homens e de envenenamento e intoxicação em mulheres $(p>0,01)$.

No mesmo período, foram notificadas 5.624 intoxicações exógenas autoprovocadas. Na tabela 2 consta a descrição dos casos e a estratificação das características por sexo. De maneira semelhante às vítimas de violência autoprovocada, prevaleceu o sexo feminino (75,3\%), idade entre 30 e 59 anos (49\%), cor da pele branca (86\%), ensino fundamental completo/incompleto (47,3\%), residência em zona urbana/periurbana $(89,6 \%)$ e ocorrência na própria residência $(96,6 \%)$. Quanto às características clínicas analisadas, o agente tóxico e a via de exposição predominantes foram, respectivamente, os medicamentos $(86,1 \%)$ e a via digestiva $(99,1 \%)$, sendo 75,2\% dos episódios caracterizados como agudos únicos, o atendimento hospitalar o mais frequente $(71,8 \%)$ e a necessidade de internação observada em $46,1 \%$ dos casos. $O$ principal critério utilizado para confirmação diagnóstica foi o clínico $(74,6 \%)$ e a classificação final mais frequente foi a intoxicação confirmada $(68,2 \%)$. Óbito consumado foi constatado em 1\% dos indivíduos ao longo da evolução e 95\% evoluiu sem qualquer sequela física.

Na distribuição das características das intoxicações conforme sexo, evidenciou-se diferença significativa de acordo com a idade, zona de residência, local de ocorrência, agente tóxico empregado, via de exposição, tipo de exposição, tipo de atendimento, necessidade de hospitalização, classificação final e evolução do caso. Mulheres nas faixas etárias inferior a 16 e entre 30 e 59 anos apresentaram frequências superiores aos homens, que predominaram nas faixas de 17 a 29 e acima dos 60 anos $(p<0,01)$. Ainda, residência em zona urbana/ periurbana e ocorrências na própria residência também foram mais frequentes em mulheres $(p=0,02 ; p<0,01)$.

Quanto aos aspectos clínicos, as prevalências de uso de medicamentos, de via digestiva de exposição e de exposições agudas repetidas foram significativamente maiores entre as mulheres $(p<0,01)$. Entre os homens predominaram o atendimento hospitalar $(p=0,03)$, a necessidade de hospitalização, a confirmação da intoxicação e o óbito $(p<0,01)$.
O número de casos autoprovocados de violência e de intoxicação, por ano, e a prevalência por 100.000 habitantes estão demonstrados na figura 1. Em ambos os agravos, notou-se aumento progressivo das notificações, tornando-se, respectivamente, 169\% e 381,1\% maiores no ano de 2017 em relação a 2013.

A tabela 3 apresenta as prevalências por CRS e por ano, exibindo maiores valores, em ambas as categorias, no ano de 2017, além de um aumento com o decorrer dos anos na maioria dos grupos. No que se refere às regiões, todas as maiores prevalências de intoxicação foram encontradas no ano de 2017, respectivamente, nas CRSs de Passo Fundo, Lajeado e Santa Cruz do Sul. Quanto às de violência autoprovocada, maiores frequências foram verificadas nas CRSS de Lajeado, nos anos de 2017 e de 2015, e de Cachoeira do Sul, em 2017.

\section{DISCUSSÃO}

A violência autoprovocada pode estar atrelada a episódios de comportamento suicida ou a ideias de autoagressão, sendo de notificação compulsória e evidenciando um importante problema de saúde pública em ambos os $\operatorname{casos}^{10}$. O cenário reflete uma sinalização de mal-estar e sofrimento psíquicos em indivíduos que não conseguem encontrar alternativas para a solução de seus conflitos, especialmente os jovens desamparados ${ }^{8}$.

Foi identificado um aumento expressivo no número de casos notificados de violências autoprovocadas e de intoxicações entre 2013 e 2017. Esse aumento foi previamente descrito por outros autores e parece refletir alguns fatores como o uso irracional de medicamentos, fácil acesso a drogas controladas, importante desenvolvimento das indústrias químicas, mas, principalmente, o esforço de políticas públicas e órgãos de saúde no incentivo à identificação e à correta notificação de casos, envolvendo também acréscimos importantes relacionados a problemas de saúde mental e transtornos psiquiátricos na população geral ${ }^{11,12}$.

O Boletim Epidemiológico emitido pelo Ministério da Saúde em 2017 revelou um crescimento nos casos de violência autoinfligida próximo a 200\% em todos os estados brasileiros, entre os anos de 2011 e 2016, convergindo com o acréscimo encontrado na amostra estudada ${ }^{6}$. No entanto, a subnotificação e os erros de classificação se mantêm frequentes e o sistema de notificação ainda é debilitado, de modo que as taxas podem ser maiores do que as relatadas ${ }^{13}$. Além disso, também a ausência dos óbitos consumados na análise ora apresentada pode contribuir para o mascaramento desses dados.

Quanto à distribuição locorregional, notaram-se maiores prevalências, em ambas as situações, nas CRSs de Lajeado, Passo Fundo, Santa Cruz do Sul e outras. Embora não possa 
Tabela 2. Casos autoprovocados de intoxicação exógena: caracterização e estratificação por sexo. Notificações no estado do Rio Grande do Sul, 2013-2017 $(n=5.624)$

\begin{tabular}{|c|c|c|c|c|}
\hline \multirow{2}{*}{ Variáveis } & Total & Masculino & Feminino & \multirow{2}{*}{$\mathbf{p}^{*}$} \\
\hline & n (\%) & n (\%) & n (\%) & \\
\hline Total & $5.624(100,0)$ & $1.387(24,7)$ & $4.237(75,3)$ & \\
\hline Idade (anos completos) & & & & $<0,01$ \\
\hline$\leq 16$ & $761(13,5)$ & $118(8,5)$ & $643(15,2)$ & \\
\hline $17-29$ & $1.900(33,8)$ & $573(41,3)$ & $1.327(31,3)$ & \\
\hline $30-59$ & $2.756(49,0)$ & $622(44,9)$ & $2.134(50,4)$ & \\
\hline$\geq 60$ & $207(3,7)$ & $74(5,3)$ & $133(3,1)$ & \\
\hline Cor de pele $(n=5.339)$ & & & & 0,10 \\
\hline Branca & $4.591(86,0)$ & $1.105(84,6)$ & $3.486(86,4)$ & \\
\hline Outras & $748(14,0)$ & $201(15,4)$ & $547(13,6)$ & \\
\hline Escolaridade $(n=4.090)$ & & & & 0,07 \\
\hline Analfabetismo & $396(9,7)$ & $100(10,3)$ & $296(9,4)$ & \\
\hline Ensino fundamental completo/incompleto & $1.936(47,3)$ & $482(49,9)$ & $1.454(46,6)$ & \\
\hline Ensino médio incompleto ou mais & $1.758(43,0)$ & $385(39,8)$ & $1.373(44,0)$ & \\
\hline Zona de residência $(n=5.488)$ & & & & 0,02 \\
\hline Urbana/periurbana & $4.916(89,6)$ & $1.184(87,9)$ & $3.732(90,1)$ & \\
\hline Rural & $572(10,4)$ & $163(12,1)$ & $409(9,9)$ & \\
\hline Local de ocorrência $(n=5.467)$ & & & & $<0,01$ \\
\hline Própria residência & $5.282(96,6)$ & $1.269(94,3)$ & $4.013(97,4)$ & \\
\hline Outros & $185(3,4)$ & $77(5,7)$ & $108(2,6)$ & \\
\hline Agente tóxico $(n=5.111)$ & & & & $<0,01$ \\
\hline Medicamento & $4.399(86,1)$ & $956(75,7)$ & $3.443(89,5)$ & \\
\hline Veneno & $414(8,1)$ & $185(14,7)$ & $229(5,9)$ & \\
\hline Outros & $298(5,8)$ & $121(9,6)$ & $177(4,6)$ & \\
\hline Via de exposição ( $\mathrm{n}=5.310)$ & & & & $<0,01$ \\
\hline Digestiva & $5.263(99,1)$ & $1.284(98,2)$ & $3.979(99,4)$ & \\
\hline Outras & $47(0,9)$ & $23(1,8)$ & $24(0,6)$ & \\
\hline Tipo de exposição (n=5.049) & & & & $<0,01$ \\
\hline Aguda, única & $3.799(75,2)$ & $989(79,8)$ & $2.810(73,8)$ & \\
\hline Aguda, repetida & $1.151(22,8)$ & $224(18,1)$ & $927(24,3)$ & \\
\hline Crônica ou aguda sobre crônica & $99(2,0)$ & $26(2,1)$ & $73(1,9)$ & \\
\hline Tipo de atendimento $(n=5.590)$ & & & & 0,03 \\
\hline Hospitalar & $4.012(71,8)$ & $1.021(74,0)$ & $2.991(71,0)$ & \\
\hline Ambulatorial ou domiciliar & $1.578(28,2)$ & $358(26,0)$ & $1.220(29,0)$ & \\
\hline Hospitalização (n=5.340) & & & & $<0,01$ \\
\hline $\operatorname{Sim}$ & $2.460(46,1)$ & $650(49,8)$ & $1.810(44,9)$ & \\
\hline Não & $3.164(53,9)$ & $656(50,2)$ & $2.224(55,1)$ & \\
\hline Classificação final ( $n=5.217)$ & & & & $<0,01$ \\
\hline Intoxicação confirmada & $3.559(68,2)$ & $917(71,2)$ & $2.642(67,3)$ & \\
\hline Exposição & $1.427(27,3)$ & $306(23,7)$ & $1.121(28,5)$ & \\
\hline Reação adversa & $108(2,1)$ & $23(1,8)$ & $85(2,2)$ & \\
\hline Diagnóstico diferencial & $68(1,3)$ & $25(1,9)$ & $43(1,1)$ & \\
\hline Outros & $55(1,1)$ & $18(1,4)$ & $37(0,9)$ & \\
\hline Critério de confirmação $(n=5.194)$ & & & & 0,17 \\
\hline Clínico & $3.876(74,6)$ & $935(73,3)$ & $2.941(75,0)$ & \\
\hline Clínico epidemiológico & $1.043(20,1)$ & $260(20,4)$ & $783(20,0)$ & \\
\hline Clínico laboratorial & $275(5,3)$ & $80(6,3)$ & $195(5,0)$ & \\
\hline Evolução do caso ( $\mathrm{n}=4.718$ ) & & & & $<0,01$ \\
\hline Cura sem sequela & $4.481(95,0)$ & $1.058(93,0)$ & $3.423(95,6)$ & \\
\hline Óbito & $84(1,8)$ & $32(2,8)$ & $52(1,5)$ & \\
\hline Outros & $153(3,2)$ & $48(4,2)$ & $105(2,9)$ & \\
\hline
\end{tabular}

* Teste do qui-quadrado.

Fonte: Sistema de Informação de Agravos de Notificação (SINAN). 
ser estabelecido como regra, as maiores taxas podem estar atreladas a um elevado crescimento socioeconômico das regiões nos últimos anos, favorecendo desigualdades socioeconômicas, e à presença de centros de saúde de referência no estado, oferecendo maior acesso à saúde e amparo em casos de ocorrências, com consequente maior número de notificações. Considerando a possibilidade de evolução da violência autoprovocada para um suicídio efetivo, um olhar regionalizado permite a identificação de espaços geográficos em que as ações preventivas podem ser enfatizadas.

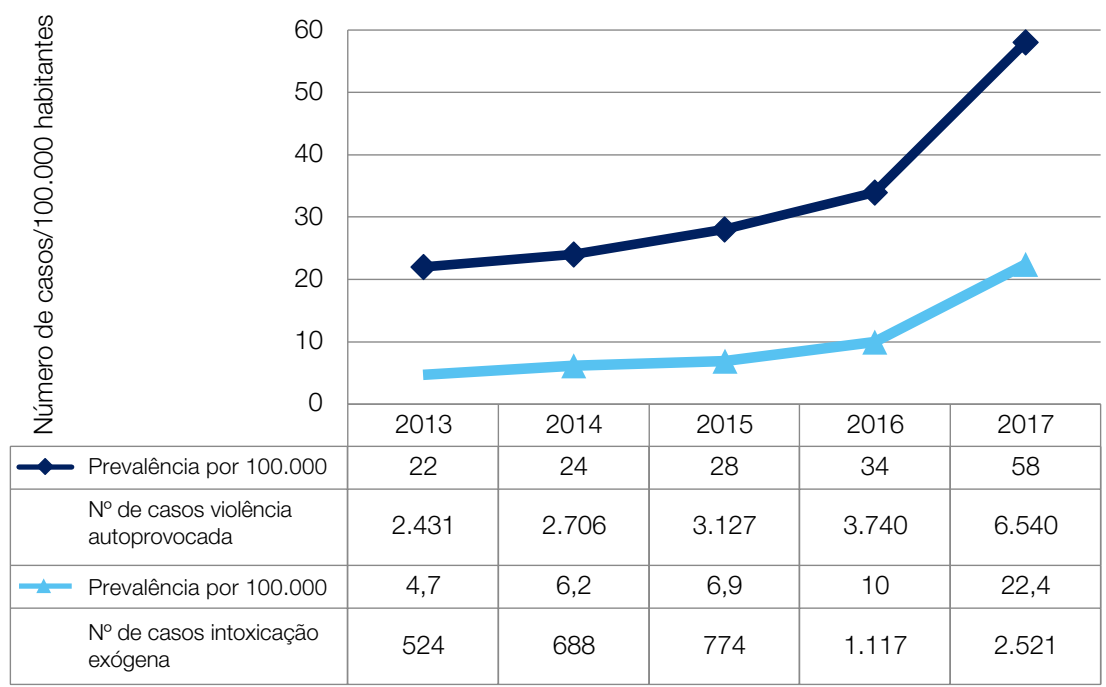

Figura 1. Violência e intoxicação exógena autoprovocada: número de casos e prevalência por 100.000 habitantes. Notificações no estado do Rio Grande do Sul, 2013-2017 ( $n=18.544 / 5.624)$. Fonte: Sistema de Informação de Agravos de Notificação (SINAN).

Tabela 3. Prevalência de casos autoprovocados de violência e de intoxicação exógena por Coordenadoria Regional de Saúde (CRS). Notificações no estado do Rio Grande do Sul, 2013-2017. Número de casos/100.000 habitantes. (n=18.544/5.620)

\begin{tabular}{|c|c|c|c|c|c|c|c|c|c|c|}
\hline \multirow[t]{2}{*}{ Ano } & \multicolumn{2}{|c|}{2013} & \multicolumn{2}{|c|}{2014} & \multicolumn{2}{|c|}{2015} & \multicolumn{2}{|c|}{2016} & \multicolumn{2}{|c|}{2017} \\
\hline & Violência & $\begin{array}{l}\text { Intoxicação } \\
\text { Exógena }\end{array}$ & Violência & $\begin{array}{l}\text { Intoxicação } \\
\text { Exógena }\end{array}$ & Violência & $\begin{array}{c}\text { Intoxicação } \\
\text { Exógena }\end{array}$ & Violência & $\begin{array}{l}\text { Intoxicação } \\
\text { Exógena }\end{array}$ & Violência & $\begin{array}{c}\text { Intoxicação } \\
\text { Exógena }\end{array}$ \\
\hline $1^{\text {a } C R S ~ P o r t o ~ A l e g r e ~}$ & 26,60 & 1,58 & 25,54 & 1,91 & 36,88 & 3,13 & 56,24 & 6,13 & 89,79 & 12,42 \\
\hline $2^{\mathrm{a}}$ CRS Porto Alegre & 1,98 & 0,18 & 1,75 & - & 2,14 & 0,55 & 5,22 & 2,75 & 12,56 & 5,85 \\
\hline $3^{\text {a }}$ CRS Pelotas & 6,38 & - & 6,25 & 0,11 & 8,16 & 0,23 & 10,64 & 5,43 & 36,25 & 9,60 \\
\hline $4^{\mathrm{a}}$ CRS Santa Maria & 40,25 & 12,11 & 49,41 & 13,33 & 38,32 & 14,19 & 29,93 & 17,18 & 47,91 & 27,40 \\
\hline $5^{\mathrm{a}}$ CRS Caxias do Sul & 34,31 & 1,14 & 38,62 & 3,99 & 39,78 & 3,53 & 43,58 & 10,34 & 67,36 & 33,60 \\
\hline $6^{\text {a }}$ CRS Passo Fundo & 63,31 & 30,89 & 57,89 & 28,33 & 49,93 & 13,66 & 61,40 & 14,97 & 99,64 & 57,73 \\
\hline $7^{\text {a }}$ CRS Bagé & 6,87 & - & 9,49 & - & 10,00 & - & 6,83 & - & 42,51 & 13,64 \\
\hline $8^{\text {a }}$ CRS Cachoeira do Sul & 41,59 & 15,96 & 39,14 & 18,85 & 56,50 & 26,56 & 66,61 & 37,16 & 107,56 & 45,34 \\
\hline $9^{a}$ CRS Cruz Alta & 14,69 & 0,64 & 21,09 & 0,64 & 11,51 & 2,56 & 22,38 & 2,56 & 67,79 & 11,51 \\
\hline $10^{\mathrm{a}} \mathrm{CRS}$ Alegrete & 17,81 & 8,59 & 20,14 & 11,33 & 29,61 & 12,39 & 26,07 & 14,92 & 80,69 & 40,19 \\
\hline $11^{\text {a }}$ CRS Erechim & 17,22 & 0,84 & 22,67 & 3,36 & 8,81 & 3,36 & 19,71 & 17,62 & 20,55 & 25,16 \\
\hline $12^{\mathrm{a}}$ CRS Santo Ângelo & 19,78 & 5,46 & 6,15 & 6,83 & 6,16 & 1,37 & 21,24 & 1,03 & 27,79 & 3,09 \\
\hline $13^{\mathrm{a}}$ CRS Santa Cruz do Sul & 11,39 & 3,21 & 25,76 & 14,14 & 57,63 & 36,20 & 60,57 & 29,13 & 105,75 & 53,45 \\
\hline $14^{\mathrm{a}}$ CRS Santa Rosa & 49,78 & 10,30 & 62,73 & 18,90 & 71,82 & 19,78 & 54,67 & 21,52 & 82,73 & 28,01 \\
\hline $15^{\mathrm{a}} \mathrm{CRS}$ Palmeira das Missões & 2,40 & - & 9,59 & 0,60 & 11,99 & 2,40 & 7,19 & 3,59 & 20,36 & 10,78 \\
\hline $16^{\text {a }}$ CRS Lajeado & 78,96 & 13,45 & 86,36 & 19,19 & 127,25 & 30,37 & 101,57 & 29,35 & 150,92 & 56,42 \\
\hline $17^{\mathrm{a}} \mathrm{CRS}$ ljuí & 6,06 & - & 24,65 & 5,62 & 19,86 & 9,07 & 54,76 & 21,56 & 75,78 & 25,83 \\
\hline 18ª CRS Osório & 11,82 & 8,80 & 27,49 & 13,61 & 24,54 & 14,56 & 27,27 & 15,51 & 55,68 & 47,46 \\
\hline $19^{\mathrm{a}}$ CRS Frederico Westphalen & 14,09 & 1,04 & 24,56 & 2,09 & 17,28 & 1,57 & 29,88 & 1,05 & 43,58 & 6,30 \\
\hline
\end{tabular}

Fonte: Sistema de Informação de Agravos de Notificação (SINAN). 
O predomínio do sexo feminino nos casos de violência autoprovocada vai ao encontro da literatura nacional e internacional, cujos dados apontam as mulheres como sendo maioria com diagnóstico de transtornos psiquiátricos ${ }^{16,14}$. É fato que a automutilação está intimamente relacionada com a depressão, transtorno duas vezes mais comum em mulheres, explicado por fatores genéticos, biológicos e neuroendócrinos associados ao gênero, mas também potencializado pelo ambiente social desigual ao que mulheres são comumente expostas, relacionado a traumas, abusos e repressões ${ }^{15,16}$. Estudo executado no Canadá demonstrou que 30\% a $40 \%$ das mulheres com histórico de abuso apresentaram episódios posteriores de distúrbio pós-traumático, depressão e tentativas de suicídio, confirmando a necessidade de considerar efeitos psicossociais cumulativos associados a eventos futuros ${ }^{16-18}$.

Enquanto mulheres planejam e tentam suicídio mais frequentemente do que os homens, eles, por sua vez, correspondem às maiores taxas de suicídio consumado ${ }^{6}$. A escolha do método parece estar bem atrelada a isso, já que mulheres costumam fazer uso de formas menos letais de autoagressão (envenenamento e ingesta medicamentosa), enquanto homens buscam métodos mais invasivos e eficazes, como enforcamento e queda de altura, padrão esse também verificado no presente estudo $0^{6,17}$.

Os padrões de idade em relação a tentativas de suicídio, violências e intoxicações autoprovocadas variam entre as regiões do mundo, estando, na maioria das vezes, relacionados a populações idosas'. Nos Estados Unidos da América, todavia, as taxas chegam a ser três vezes maiores entre adultos jovens de 18 a 25 anos $^{18}$. Segundo o Boletim Epidemiológico de 2017, o Brasil apresenta predomínio de vítimas com idade entre 10 e 29 anos em ambos os fenômenos e, da mesma forma, estudo realizado com análise dos suicídios e das tentativas de suicídio na cidade de Uberaba, Minas Gerais, mostrou predomínio de jovens com idades de 20 a 29 anos a,19. $^{6}$. Entretanto, no presente estudo, foi encontrada maior prevalência entre pessoas de 30 a 59 anos (46,4\%), possivelmente justificada pela maior frequência de mulheres em idade reprodutiva, que correspondem a cerca de $70 \%$ das vítimas, compondo uma "janela de vulnerabilidade" muito relacionada a maiores exposições, pressões e cargas sociais ${ }^{16,20}$.

Sabe-se, classicamente, que a população do Rio Grande do Sul apresenta cor de pele majoritariamente branca (cerca de $80 \%$ dos indivíduos), e tal dado corrobora a elevada prevalência da etnia nos casos notificados. Quando comparados com dados da literatura nacional sobre o tema, contudo, há discordâncias, seja pela heterogeneidade das populações avaliadas ou pela tendência ao preenchimento errôneo da variável raça nas fichas de notificações ${ }^{6}$. No caso da baixa escolaridade, já é bem descrita como um fator de risco para a violência autoprovocada $a^{6,14}$.
Ainda, constatou-se que 90\% dos casos de violência ocorreram na própria residência do indivíduo e em zona urbana/periurbana, com diferença estatisticamente significativa em relação ao sexo e maiores frequências nas mulheres. Tais observações se assemelham às apresentadas na literatura, em que o local de ocorrência é justificado pela facilidade de acesso aos meios empregados, exemplificados pelos medicamentos e raticidas ${ }^{6,14}$.

No que se refere às intoxicações exógenas, são descritas como o método mais comumente utilizado em casos de violência autoinfligida e, consequentemente, em tentativas de suicídio ${ }^{12,13}$. Todavia, são consideradas um agravo evitável, corroborando que estratégias preventivas voltadas à saúde mental podem contribuir para a diminuição dos casos na população6.

Averiguou-se que três em cada quatro episódios de intoxicação notificados se deram em mulheres, achados que coincidem com os de outros estados e cidades brasileiras, os quais apontam o sexo feminino mais frequentemente em tentativas de suicídio e o masculino em suicídios consumados ${ }^{8,11,21-25}$. As diferenças advêm, entre outros fatores, da preferência pelo uso de substâncias entre as mulheres, da maior exposição a vulnerabilidades diante de estresses psicossociais e da maior predição ao desenvolvimento de psicopatologias, enquanto homens tendem a adotar o uso de técnicas mais agressivas, que usualmente levam ao suicídio consumado, além de estarem mais associados a impulsividades, instabilidades de humor e demora na busca por ajuda, contribuindo para o desfecho fata|8,26.

Quanto à idade, notou-se maior frequência de intoxicações na faixa entre 30 e 59 anos (49\%), embora mulheres apresentem frequências ligeiramente maiores quando abaixo dos 16 anos. Dados semelhantes já foram descritos e convergem com as tendências globais, que colocam o suicídio como a segunda ou a terceira causa de morte em indivíduos entre 15 e 34 anos e cerca de 10 tentativas relatadas para cada suicídio consumado ${ }^{25,27}$. O maior número de casos parece estar relacionado a um maior acesso a medicamentos e outros agentes intoxicantes nessa faixa, demonstrando que estratégias para a redução desse fator poderiam ser eficientes na prevenção do agravo ${ }^{25}$.

Quanto ao perfil sociodemográfico, notou-se que menor escolaridade pode estar relacionada a menor poder socioeconômico e importantes prejuízos à qualidade de vida, enquanto a ocorrência na residência remete a um íntimo componente familiar, ${ }^{8,2}$. Foi encontrada, ainda, diferença significativa entre o sexo e características inerentes às vítimas, comprovando, especialmente, a associação entre homens e quadros mais agravados (necessidade de hospitalização, desfechos fatais e intoxicações confirmadas) e entre mulheres e episódios repetidos ou ocorridos na própria residência, contudo essas últimas ainda não são profundamente 
estudadas na literatura e são destacadas como uma lacuna no tema ${ }^{29}$.

Referente ao agente tóxico empregado, as mulheres se sobressaíram no uso de medicamentos (78,3\%), embora a frequência entre sexos se mostre pouco diferente quanto aos demais agentes. Estudos demonstram que as principais substâncias utilizadas nos países desenvolvidos são, respectivamente, os medicamentos e os agrotóxicos, com inversão da primeira posição nos menos desenvolvidos ${ }^{22}$. Embora o Brasil não esteja entre os países desenvolvidos, outros estudos regionais também mostram maior frequência do uso de medicamentos em tentativas de suicídio por intoxicação exógena, indicando que esse é o método escolhido pela maioria dos brasileiros que buscam o autoextermínio 8,12,21,26,30. O resultado é condizente com levantamentos que apontam os medicamentos como método com maior disponibilidade, aceitabilidade social e preferência em ambos os sexos, mas com menor tendência de evolução para fatalidade ${ }^{26}$.

Ainda no panorama das exposições, evidenciou-se um maior número de ocorrências agudas únicas por via digestiva, semelhantes aos observados em outros serviços de urgência e emergência ${ }^{8}$. Em relação ao atendimento, foi previamente estabelecido na literatura que quase a totalidade dos pacientes tende a evoluir sem qualquer tipo de sequela. Contudo, vale citar que os casos notificados refletem apenas as intoxicações que demandam assistência hospitalar e que não evoluem a óbito previamente, perdendo-se informações acerca dos casos de menor e maior gravidade que não chegam ao serviço $0^{8,11,28,30}$. Ainda, possíveis subnotificações e mal preenchimento das fichas podem ser determinantes para uma dificuldade na obtenção de dados que retratem o comportamento do uso autoprovocado de agentes tóxicos nas populações.

Cabe destacar que a dependência do correto preenchimento das fichas de notificação, para eficiente análise dos dados, configura-se como a principal limitação do estudo, já que a subnotificação, o preenchimento incompleto ou inadequado e a incorreta classificação dos episódios podem dificultar uma real descrição epidemiológica dos agravos. Ainda, a impossibilidade do contato direto com as vítimas e da busca ativa dos dados nas unidades responsáveis pelo atendimento impede uma maior elucidação dos resultados ora apresentados.

\section{CONCLUSÕES}

Assim, evidencia-se um aumento no número de casos autoprovocados de violência e de intoxicação no estado do Rio Grande do Sul no período analisado, indicando um aumento na disponibilidade dos meios de autoagressão, mas também um acréscimo no surgimento de novos casos de transtornos psiquiátricos na população, potencialmente associados a um contexto psicossocial. Importantes diferenças de acordo com o sexo das vítimas foram encontradas e podem servir de subsídio para futuras pesquisas, práticas clínicas e, sobretudo, na implantação de medidas preventivas. Espera-se, por fim, que este trabalho possa contribuir com a comunidade científica de modo a incentivar ações e políticas no âmbito da gestão em saúde para prevenção e implementação de medidas contra o comportamento suicida ou autoagressivo, bem como para a correta identificação e manejo adequado dos casos.

\section{CONTRIBUIÇÕES INDIVIDUAIS}

Luis Felipe Chaga Maronezi - Contribuiu na concepção e desenho do estudo, na análise estatística, interpretação de dados, elaboração do artigo e aprovação da versão final a ser publicada.

Giovana Bonessoni Felizari - Contribuiu na análise estatística, interpretação de dados, elaboração do artigo e aprovação da versão final a ser publicada.

Guilherme Assoni Gomes - Contribuiu na concepção e desenho do estudo, revisão crítica do artigo e aprovação da versão final a ser publicada.

Jeanice de Freitas Fernandes - Contribuiu na concepção e desenho do estudo, revisão crítica do artigo e aprovação da versão final a ser publicada.

Rogério Tomasi Riffel - Contribuiu na análise estatística, interpretação de dados, revisão crítica do artigo e aprovação da versão final a ser publicada.

Ivana Loraine Lindemann - Contribuiu na concepção e desenho do estudo, na análise estatística, interpretação de dados, revisão crítica do artigo e aprovação da versão final a ser publicada.

\section{CONFLITOS DE INTERESSE}

Financiamentos: O primeiro autor declara ter recebido, durante 12 meses, bolsa financiada pelo Edital CNPq - PIBIC: 658/GR/UFFS/2019, para participação no estudo. O segundo autor declara ter recebido, durante 12 meses, bolsa financiada pelo Edital PIBIC: 459/GR/UFFS/2019, para participação no estudo.

Todos os autores declaram não ter quaisquer conflitos de interesse com a realização do estudo.

\section{AGRADECIMENTOS}

Em especial, ao Centro Estadual de Vigilância em Saúde - Núcleo de Vigilância das Doenças e Agravos Não Transmissíveis, pelo suporte e disponibilização dos dados solicitados. 


\section{REFERÊNCIAS}

1. World Health Organization (WHO). Preventing suicide: a global imperative. Geneva: WHO; 2014. 89p.

2. Spiller HA, Appana S, Brock GN. Epidemiological trends of suicide and attempted suicide by poisoning in the US: 2000-2008. Leg Med. 2010;12(4):177-83.

3. World Health Organization (WHO). The World health report 2003: shaping the future. Geneva: WHO; 2003. 193p.

4. Almeida TSO, Fook SML, MarizSR, Camêlo ELS, Gomes LCF. Suicide attempts: epidemiologic trends towards geoprocessing. Ciênc Saúde Coletiva. 2018;23(4):1183-92.

5. Conte M, Cruz CW, Silva CG, Castilhos NRM, Nicolella ADR. Encontros ou Desencontros: histórias de idosos que tentaram suicídio e a Rede de Atenção Integral em Porto Alegre/RS, Brasil. Ciênc Saúde Coletiva. 2015;20(6):1741-9.

6. Brasil. Ministério da Saúde. Secretaria de Vigilância em Saúde. Perfil epidemiológico das tentativas e óbitos por suicídio no Brasil. Boletim Epidemiológico. 2017;48(30).

7. Brasil. Ministério da Saúde. Secretaria de Vigilância em Saúde. Perfil epidemiológico dos casos notificados de violência autoprovocada e óbitos por suicídio entre jovens de 15 a 29 anos no Brasil, 2011 a 2018. Boletim Epidemiológico. 2019;50(24).

8. Veloso C, Monteiro CFS, Veloso LUP, Figueiredo MLF, Fonseca RSB, Araújo TME, et al. Violência autoinfligida por intoxicação exógena em um serviço de urgência e emergência. Rev Gaucha Enferm. 2017;38(2)

9. Brasil. Ministério da Saúde. Secretaria de Vigilância em Saúde. Departamento de Vigilância em Saúde Ambiental e Saúde do Trabalhador. Instruções para preenchimento da Ficha de Investigação de Intoxicação Exógena Sinan (Sistema de Informação de Agravos de Notificação). Brasília, DF: Ministério da Saúde; 2018. 42 p, 1 v.

10. Brasil. Ministério da Saúde. Secretaria de Vigilância em Saúde. Coordenação Geral de Desenvolvimento da Epidemiologia em Serviços. Guia de vigilância em Saúde. 2a ed. Brasília, DF: Ministério da Saúde; 2017.

11. Timóteo MVF, Araujo FJR, Sampaio FAF, Pereira TAC, Torres DSB, Martins KCP, et al. Panorama das intoxicações associadas ao uso de medicamentos registradas no Brasil. Res Soc Dev. 2020;9(4)

12. Toscano MM, Landim JTA, Rocha AB, Sousa-Muñoz RL. Intoxicações exógenas agudas registradas em centro de assistência toxicológica. Rev Saúde Pesq. 2016;9(3):425-32.

13. Marcolan JF. For a public policy of surveillance of suicidal behavior. Rev Bras Enferm. 2018;71(Suppl 5):2343-7.

14. Bahia CA, Avanci JQ, Pinto LW, Minayo MCS. Lesão autoprovocada em todos os ciclos da vida: perfil das vítimas em serviços de urgência e emergência de capitais do Brasil. Ciênc Saúde Coletiva. 2017;22(9):2841-50

15. Park LT, Carlos AZ. Depression in the Primary Care Setting. N Engl J Med. 2019;(380):559-68.
16. Lemieux S, Tourigny M, Joly J, Baril K, Séguin M. Caractéristiques associées à la dépression et aux symptômes de stress post-traumatique chez les femmes victimes d'agression sexuelle durant l'enfance. Revue d'Épidémiologie et de Santé. 2019.

17. Gomes R, Nascimento EF, Araújo FC. Por que os homens buscam menos os serviços de saúde do que as mulheres? As explicações de homens com baixa escolaridade e homens com ensino superior. Cad Saúde Pública. 2007;23(3):565-74.

18. Centers for Disease Control and Prevention. Web-based Injury Statistics Query and Reporting System (WISQARS). Leading Causes of Death Reports 1981-2016. Atlanta, GA. U.S. Department of Health and Human Services.

19. Ribeiro NM, Castro SS, Scatena LM, Haas VJ. Análise da tendência temporal do suicídio e de sistemas de informações em saúde em relação às tentativas de suicídio. Texto Contexto Enferm. 2018;27(2)

20. O'Rourke MC, Jamil RT, Siddiqui W. Suicide Screening and Prevention. StatPearls Publishing. 2021.

21. Monteiro RA, Bahia CA, Paiva EA, Sá NNB, Minayo MCS. Hospitalizações relacionadas a lesões autoprovocadas intencionalmente: Brasil, 2002 a 2013. Ciênc Saúde Coletiva. 2015;20(3):689-700.

22. Santos SA, Legay LF, Lovisi GM, Santos JFC, Lima LA. Suicídios e tentativas de suicídios por intoxicação exógena no Rio de Janeiro: análise dos dados dos sistemas oficiais de informação em saúde, 2006-2008. Rev Bras Epidemiol. 2013;16(2):376-87.

23. Bordoni PHC, Nascentes ALM, Caixeta LJ, Reis LS, Luiz LFR, Gomes LPB, et al. Estudo transversal das necropsias de intoxicação exógena do Instituto Médico-Legal de Belo Horizonte no período 2006-2012. Brazilian Journal of Forensic Sciences, Medical Law and Bioethics. 2017;6(3):359-77.

24. Maia SS, Souza VS, Souza ED, Faustino TN. Anos Potenciais de Vidas Perdidos por intoxicação exógena, de 2007 a 2017, no Brasil. Rev Enferm Contemporânea. 2019;8(2):135-42.

25. Vieira LP, Santana VTP, Suchara EA. Caracterização de tentativas de suicídios por substâncias exógenas. Cad Saúde Coletiva. 2015;23(2):118-23.

26. Santos SA, Legay LF, Lovisi GM. Substâncias tóxicas e tentativas e suicídios: considerações sobre acesso e medidas restritivas. Cad Saúde Coletiva. 2013;21(1):53-61.

27. Gonçalves $L R C$, Gonçalves $E$, Oliveira Júnior $L B$. Determinantes espaciais e socioeconômicos do suicídio no Brasil: uma abordagem regional. Nova Economia. 2011;21(2):281-316.

28. Oliveira EN, Félix TA, Mendonça CBL, Souza DR, Ferreira GB, Freire MA, et al. Tentativa de suicídio por intoxicação exógena: contexto de notificações compulsórias. Revista Eletrônica Gestão \& Saúde. 2015;6(3):2497-511.

29. Vieira NRS, Dantas RAN, Dantas DV, Santos JJS, Vasconcelos EFL, Carvalho ICT. Caracterização da produção científica sobre intoxicações exógenas: revisão integrativa da literatura. Rev Saúde. 2016;10(1-2):47-60.

30. Hahn RC, Camargo MP, Oliveira MLF. Características de intoxicações agudas em crianças: estudo em um centro de assistência toxicológica. Braz J Surg Clin Res. 2013;4(1):18-22. 